\title{
THE DOWNWARD DIRECTED GROUNDS HYPOTHESIS AND VERY LARGE CARDINALS
}

\author{
TOSHIMICHI USUBA
}

\begin{abstract}
A transitive model $M$ of ZFC is called a ground if the universe $V$ is a set forcing extension of $M$. We show that the grounds of $V$ are downward setdirected. Consequently, we establish some fundamental theorems on the forcing method and the set-theoretic geology. For instance, (1) the mantle, the intersection of all grounds, must be a model of ZFC. (2) $V$ has only set many grounds if and only if the mantle is a ground. We also show that if the universe has some very large cardinal, then the mantle must be a ground.
\end{abstract}

\section{INTRODUCTION}

After Cohen's solution of the continuum hypothesis, the forcing method became an important tool of set-theory. While many independent results are proved by the forcing method, the nature of forcing is also a fundamental topic in this field.

The forcing method uses second order objects, ground models, and generic extensions. A natural framework for treating such objects is a second-order set theory such as Von Neumann-Bernays-Gödel set-theory. However in the first order set theory ZFC, it is more difficult to treat. On the other hand, Laver [10] and, independently, Woodin found that a ground model is definable in its set-forcing extension. Later, Fuchs-Hamkins-Reitz [4] refined Laver and Woodin's result, and they give a uniform definition of all ground models of the universe:

Theorem 1.1 (Reitz [12], Fuchs-Hamkins-Reitz [4]). There is a first order formula $\varphi(x, r)$ of set theory such that:

(1) For each $r$, the class $W_{r}=\{x: \varphi(x, r)\}$ is a ground model of $V$.

(2) For every ground model $M$ of $V$, there is $r$ such that $M=W_{r}$.

This allows us to treat ground models within the first order set theory ZFC. Using this uniform definition, a study of the structure of all ground models was initiated, which attempts a study of the nature of the forcing method. The study is now called the set-theoretic geology. In [4], some notions in the forcing method and the set-theoretic geology were introduced. One of the most important and natural concepts is the notion of the mantle:

2010 Mathematics Subject Classification. Primary 03E40, 03E45, 03E55.

Key words and phrases. Forcing method, Set-theoretic geology, Downward directed grounds hypothesis, Large cardinal, Generic multiverse. 
Definition 1.2. The mantle is the intersection of all ground models of the universe.

The mantle is a very natural and canonical object in the context of the forcing method. Fuchs-Hamkins-Reitz [4] proved the structure of the mantle can be manipulated by class forcings. For example, they proved that the universe $V$ can be the mantle of some class forcing extension $V[G]$. Besides the natural definition of the mantle, there are many open questions about the mantle. An important one is whether the mantle is a model of $\mathrm{ZFC}$ or not. If it is a model of $\mathrm{ZFC}$, then the mantle can be seen as the core of all ground models.

The key to the solution of this question is the downward directedness of the ground models. The downward directed grounds hypothesis, DDG, is the assertion that every two ground models have a common ground model. The strong DDG is the assertion that every collection $\left\{W_{r}: r \in X\right\}$ of ground models indexed by some set $X$ has a common ground model. In [4], it was proved that if DDG holds, then the mantle is a model of ZF. Furthermore, if the strong DDG holds, then the mantle satisfies the axiom of choice. The DDG is not only a key to the solution, but it expresses a fundamental property of the structure of ground models.

All known models, such as $L[A], K, \mathrm{HOD}$, and class forcing extensions, satisfy the strong DDG, and their mantles are models of ZFC. So one might expect that the DDG is a theorem of ZFC. Meanwhile, attempts have been made to construct a counterexample by the forcing method or inner model construction.

In this paper, we prove that the strong DDG is actually a theorem of ZFC.

Theorem 1.3. The strong DDG holds.

Because of this result, under ZFC, some fundamental properties of the settheoretic geology can be derived without any assumptions. For example, we prove the following:

Theorem 1.4. (1) The mantle is a model of $Z F C$.

(2) The universe $V$ has only set many ground models if and only if the mantle is the minimum ground of all forcing extensions of $V$.

Next we look at the statement that " $V$ has class many ground models". A natural model of this statement is given by a class Easton forcing extension, and one can show that this statement is consistent with supercompact cardinals as follows. For a cardinal $\kappa$, by restricting the domain of Easton forcing, we may assume that a class Easton forcing is $\kappa$-directed closed. Combining this observation with Laver's indestructiblity theorem ([9]), we have the consistency of the statement that " $V$ has class many ground models" + "there exists a supercompact cardinal".

In contrast to supercompact cardinals, it is known that some very large cardinals, such as extendible cardinals, cannot be indestructible for directed closed forcing (Bgaria-Hamkins-Tsaprounis-Usuba [1]), so we cannot prove the consistency of 
" $V$ has class many ground models" + "there exists an extendible cardinal" by this method. Hence it is natural to ask whether existence of class many ground models is consistent with very large cardinals. In the last half of this paper, we will give a partial answer to this question, we show that some very large cardinal is inconsistent with class many ground models.

Definition 1.5. Let $\kappa$ be an infinite cardinal. We say that $\kappa$ is hyper-huge if for every $\lambda>\kappa$, there is an elementary embedding $j: V \rightarrow M$ into some inner model $M$ such that the critical point of $j$ is $\kappa, \lambda<j(\kappa)$, and ${ }^{j(\lambda)} M \subseteq M$.

We show that our hyper-huge cardinal excludes class many ground models. Furthermore, if a hyper-huge cardinal exists, then the mantle must be the minimum ground model of the universe. This indicates an unexpected connection between large cardinal axioms and the forcing method.

Theorem 1.6. Suppose a hyper-huge cardinal exists. Then the universe $V$ has only set many ground models, and the mantle is a ground model of $V$.

In the last section, we show some interesting consequences of main theorems, and will prove some fundamental properties about set-theoretic geology and the generic multiverse.

\section{BASIC MATERIALS}

In this section, we present some basic notations, definitions, and known facts. We also make some easy observations.

Throughout this paper, $V$ will denote the universe, so $V$ is a transitive model of ZFC containing all ordinals unless otherwise specified.

Note 2.1. In this paper, a class means a second order object in the sense of Von Neumann-Bernays-Gödel set-theory unless otherwise specified. We do not require that a class $M$ is definable in $V$ with some parameters, but we assume that $V$ satisfies the replacement schemes for the formulas of the language $\{\in, M\}$ (where we identify $M$ as a unary predicate). Note that, for every definable class $M, V$ satisfies the replacement schemes for the formulas of the language $\{\in, M\}$. Hence, every definable class is a class in our sense. Also note that if $W$ is a ground model of $V$, then $W$ is a class in our sense.

In this paper, we will assume that a transitive model $M \subseteq V$ of $\mathrm{ZF}(\mathrm{C})$ is a class of $V$ unless otherwise specified.

See Theorem 13.9 in Jech [7], and see Definition 13.2 in [7] for the definition of the Gödel operations.

Fact 2.2. Let $N \subseteq V$ be a transitive class containing all ordinals. Then $N$ is a model of ZF if and only if $N$ is closed under the Gödel operations and $N$ is almost universal, that is, for every set $x \subseteq N$, there is $y \in N$ with $x \subseteq y$. 
Note that the replacement schemes for the formulas of the language $\{\in, N\}$ is needed to prove this fact.

By Fact 2.2, for a transitive class $N \subseteq V$ containing all ordinals, the statement " $N$ is a model of ZFC" is an abbreviation of the first order formula of the language $\{\in, N\}$ that " $N$ is closed under the Gödel operations" + " $N$ is almost universal" + " $N$ satisfies the axiom of choice".

For a transitive model $M$ of ZFC and an ordinal $\alpha$, let $M_{\alpha}$ be the set of all $x \in M$ with rank $<\alpha$.

We discuss the consistency strength of a hyper-huge cardinal. Recall that an infinite cardinal $\kappa$ is 2-huge if there exists a transitive model $N$ of ZFC and an elementary embedding $j: V \rightarrow N$ with critical point $\kappa$ such that $N$ is closed under $j(j(\kappa))$-sequences.

Lemma 2.3. Suppose $\kappa$ is 2-huge. Then $V_{\kappa}$ is a model of $Z F C+$ "there are proper class many hyper-huge cardinals". Hence the consistency strength of the existence of a hyper-huge cardinal is strictly weaker than of the existence of a 2-huge cardinal.

Proof. Let $j: V \rightarrow N$ be a 2-huge embedding with critical point $\kappa$. First we check that $V_{j(\kappa)}$ is a model of " $\kappa$ is hyper-huge". To see this, take $\lambda$ with $\kappa<\lambda<j(\kappa)$.

Now we let $(\kappa, \lambda) \rightarrow\left(\kappa^{\prime}, \lambda^{\prime}\right)$ be the assertion that there is an elementary embedding $j: V \rightarrow M$ for some $M$ such that:

(1) the critical point of $j$ is $\kappa$,

(2) $\lambda<j(\kappa)=\kappa^{\prime}$,

(3) $\lambda^{\prime}=j(\lambda)$, and

(4) $M$ is closed under $\lambda^{\prime}$-sequences.

Note that $(\kappa, \lambda) \rightarrow\left(\kappa^{\prime}, \lambda^{\prime}\right)$ is equivalent to the existence of a normal ultrafilter $U$ over $\mathcal{P}\left(\lambda^{\prime}\right)$ with $\left\{x \subseteq \lambda^{\prime}: x \cap \kappa \in \kappa\right.$, ot $\left(x \cap \kappa^{\prime}\right)=\kappa$, ot $\left.(x)=\lambda\right\} \in U$.

Since $j$ is a 2-huge embedding and $\lambda<j(\kappa)$, we have $j(\lambda)<j(j(\kappa))$ and $(\kappa, \lambda) \rightarrow$ $(j(\kappa), j(\lambda))$. Now $\kappa$ is $j(\kappa)$-supercompact in $V$, hence $j(\kappa)$ is $j(j(\kappa))$-supercompact in $N$, actually in $V . j(\kappa)<j(\lambda)<j(j(\kappa))$, hence we have there is some $\kappa^{\prime}, \lambda^{\prime}<$ $j(\kappa)$ with $(\kappa, \lambda) \rightarrow\left(\kappa^{\prime}, \lambda^{\prime}\right)$ in $V$ by a standard reflection argument using the $j(j(\kappa))$ supercompactness of $j(\kappa)$. This shows that $\kappa$ is hyper-huge in $V_{j(\kappa)}=N_{j(\kappa)}$. By the elementarity of $j$, we have that $\left\{\alpha<\kappa: \alpha\right.$ is hyper-huge in $\left.V_{\kappa}\right\}$ is unbounded in $\kappa$.

A poset $\mathbb{P}$ is weakly homogeneous if, for every $p, q \in \mathbb{P}$, there is an automorphism $f$ on $\mathbb{P}$ such that $f(p)$ is compatible with $q$.

For an infinite cardinal $\chi$, let $\mathcal{H}(\chi)$ be the set of all sets $x$ such that the transitive closure of $x$ has cardinality $<\chi$. It is known that if $\chi$ is regular uncountable, then $\mathcal{H}(\chi)$ is a transitive model of $\mathrm{ZFC}-\mathrm{P}$, ZFC minus the power set axiom, with $\chi \subseteq \mathcal{H}(\chi)$. 
Here we recall some definitions and facts about set-theoretic geology. See FuchsHamkins-Reitz [4] for more information.

Definition 2.4. A transitive model $M \subseteq V$ of ZFC is a ground of $V$ if there exists a poset $\mathbb{P} \in M$ and an $(M, \mathbb{P})$-generic filter $G \subseteq \mathbb{P}$ with $M[G]=V$.

Note that $V$ is a ground of $V$ by the trivial forcing.

It is known that all grounds can be defined uniformly.

Fact 2.5 (Reitz [12], Fuchs-Hamkins-Reitz [4]). There is a first order formula $\varphi(x, r)$ of set-theory such that:

(1) For each $r$, the (definable) class $W_{r}=\{x: \varphi(x, r)\}$ is a ground of $V$.

(2) For every ground $M$ of $V$, there is $r$ such that $M=W_{r}$.

For this formula $\varphi$, the collection $\left\{W_{r}: r\right.$ is a set $\}$ consists of the grounds of $V$.

Definition 2.6. The downward directed grounds hypothesis, DDG, is the assertion that, the grounds are downward directed, that is, for every $r$ and $s$, there is $t$ such that $W_{t} \subseteq W_{r} \cap W_{s}$. The strong DDG is the assertion that, for every set $X$, there is $s$ such that $W_{s} \subseteq \bigcap_{r \in X} W_{r}$.

Next we explicitly define how to count the grounds.

Definition 2.7. We say that $V$ has only set many grounds if there is a set $X$ such that the collection $\left\{W_{r}: r \in X\right\}$ consists of the grounds of $V$, that is, for every $s$, there is $r \in X$ with $W_{r}=W_{s}$. If there is no such $X$, then $V$ has proper class many grounds. If $|X|<\kappa(\leq \kappa$, respectively), then $V$ has only $<\kappa$ many grounds ( $\kappa$ many grounds, respectively).

Definition 2.8 (Fuchs-Hamkins-Reitz [4]). The mantle, denoted by $\mathbb{M}$, is the class $\bigcap\left\{W_{r}: r\right.$ is a set $\}$.

The mantle is transitive and contains all ordinals. We will show that the mantle must be a model of ZFC.

Note 2.9. If a poset $\mathbb{P}$ is weakly homogeneous and $G$ is $(V, \mathbb{P})$-generic, then the mantle of $V[G], \mathbb{M}^{V[G]}$, is the same as the class $\left\{x \in V: \Vdash_{\mathbb{P}}\right.$ " $\left.x \in \mathbb{M}^{V[\dot{G}] "}\right\}$ defined in $V$ (where $\dot{G}$ is the canonical name for the generic filter). Hence we can let $\mathbb{M}^{\mathbb{P}}$ denote the mantle of a forcing extension of $V$ by $\mathbb{P}$.

Definition 2.10 (Fuchs-Hamkins-Reitz [4]). The generic mantle, denoted by $g \mathbb{M}$, is the class $\bigcap\left\{\mathbb{M}^{V^{\operatorname{Col}(\theta)}}: \theta\right.$ is an ordinal $\}$.

Fact $2.11([4]) . \quad$ (1) $g \mathbb{M}$ is the intersection of all grounds of all forcing extensions of $V$.

(2) $g \mathbb{M}$ is a transitive model of ZF containing all ordinals

(3) $g \mathbb{M}$ is a forcing invariant class: for every forcing extension $V[G]$ of $V$, we have $g \mathbb{M}^{V}=g \mathbb{M}^{V[G]}$. 


\section{GRounds And GENERIC EXTENSIONS}

In this section, we discuss some basic properties of grounds and generic extensions.

The following useful fact is well-known. See Lemma 15.43 in Jech [7] for the proof.

Fact 3.1. Let $M, N$ be transitive models of $Z F C$. If there is a poset $\mathbb{P} \in M$ and an $(M, \mathbb{P})$-generic $G$ with $M \subseteq N \subseteq M[G]$, then there is a complete suborder $\mathbb{Q} \subseteq \operatorname{ro}(\mathbb{P})^{M}$ in $M$ and an $(M, \mathbb{Q})$-generic $H$ such that $M[H]=N$, and $G$ is $\left(N, \operatorname{ro}(\mathbb{P})^{M} / H\right)$-generic with $M[G]=N[G]$, where $\operatorname{ro}(\mathbb{P})^{M}$ is the completion of $\mathbb{P}$ in $M$. In particular, $M$ is a ground of $N$, and $N$ is a ground of $M[G]$.

We define the covering and the approximation properties, which are important tools to investigate grounds and generic extensions.

Definition 3.2 (Hamkins [5]). Let $M \subseteq V$ be a transitive model of ZFC containing all ordinals. Let $\kappa$ be an infinite cardinal.

(1) We say that $M$ satisfies the $\kappa$-covering property for $V$ if, for every set $A$ of ordinals with $|A|<\kappa$, there is a set $B \in M$ of ordinals such that $|B|<\kappa$ and $A \subseteq B$.

(2) We say that $M$ satisfies the $\kappa$-approximation property for $V$ if, for every set $A$ of ordinals, if $A \cap x \in M$ for every set $x \in M$ of ordinals with $|x|<\kappa$, then $A \in M$.

Note 3.3. (1) $M$ satisfies the $\kappa$-covering property for $V$ if and only if, for every set $x \subseteq M$ with size $<\kappa$, there is $y \in M$ such that $x \subseteq y$ and $|y|<\kappa$.

(2) $M$ satisfies the $\kappa$-approximation property for $V$ if and only if, for every set $A \subseteq M$, if $A \cap x \in M$ for every set $x \in M$ with $|x|<\kappa$ then $A \in M$.

The following fact is key to the definition of the grounds as in Fact 2.5. See Laver [10] for a proof, and in Lemma 4.3, we will redo Laver's proof for models of $\mathrm{ZFC}-\mathrm{P}$.

Fact 3.4 (Hamkins). Let $\kappa$ be an uncountable cardinal. For transitive models $M, N \subseteq V$ of ZFC containing all ordinals, suppose $M$ and $N$ satisfy the following properties:

(1) $M, N$ satisfy the $\kappa$-covering and the $\kappa$-approximation properties for $V$.

(2) $\mathcal{P}(\kappa)^{M}=\mathcal{P}(\kappa)^{N}$.

(3) $\left(\kappa^{+}\right)^{M}=\left(\kappa^{+}\right)^{N}=\kappa^{+}$.

Then $M=N$.

The following is Lemma 13 in Hamkins [5] (and see also Mitchell [11]). 
Fact 3.5. Suppose $M$ is a ground of $V$, and $M[G]=V$ for some $\mathbb{P} \in M$ and $(M, \mathbb{P})$-generic $G$. Let $\kappa$ be an infinite cardinal. If $|\mathbb{P}|^{M}<\kappa$, then $M$ satisfies the $\kappa$-covering and the $\kappa$-approximation properties for $V$.

Lemma 3.6. Suppose $M, N$ are grounds of $V$. Let $\mathbb{P} \in M \cap N$ be a poset, $G$ be an $(M, \mathbb{P})$-generic, and $H$ be an $(N, \mathbb{P})$-generic such that $V=M[G]=N[H]$. If $\lambda=|\mathbb{P}|^{M}=|\mathbb{P}|^{N}$ and $\mathcal{P}(\lambda)^{M}=\mathcal{P}(\lambda)^{N}$, then $M=N$.

Proof. Let $\kappa=\lambda^{+}$. Note that $\kappa=\left(\lambda^{+}\right)^{M}=\left(\lambda^{+}\right)^{N}$. Moreover $\left([\kappa]^{<\kappa}\right)^{M}=\left([\kappa]^{<\kappa}\right)^{N}$ because $\mathcal{P}(\lambda)^{M}=\mathcal{P}(\lambda)^{N}$.

In order to prove $M=N$, by Fact 3.4, it is sufficient to check that the conditions (1)-(3) in Fact 3.4 hold for $M$ and $N$. (1) follows from Fact 3.6, and (3) is clear.

For (2), we prove $\mathcal{P}(\kappa)^{M} \subseteq \mathcal{P}(\kappa)^{N}$. The converse direction follows from the same argument. Fix $A \in \mathcal{P}(\kappa)^{M}$. By the $\kappa$-approximation property of $N$, it is sufficient to see that $A \cap x \in M$ for every $x \in\left([\kappa]^{<\kappa}\right)^{M}$, and this is immediate because $\left([\kappa]^{<\kappa}\right)^{M}=\left([\kappa]^{<\kappa}\right)^{N}$.

Next we define a strong version of the covering property.

Definition 3.7. Let $\kappa$ be an infinite cardinal, and $M \subseteq V$ be a transitive model of ZFC containing all ordinals. We say that $M$ satisfies the $\kappa$-uniform covering property $^{1}$ for $V$ if, for every ordinal $\alpha$ and every function $f: \alpha \rightarrow O N$, there is a function $F \in M$ such that $\operatorname{dom}(F)=\alpha, f(\beta) \in F(\beta)$ and $|F(\beta)|<\kappa$ for all $\beta<\alpha$.

Note 3.8. Suppose $M$ satisfies the $\kappa$-uniform covering property for $V$.

(1) For every cardinal $\lambda>\kappa, M$ satisfies the $\lambda$-uniform covering property for $V$.

(2) If $\kappa$ is regular, then $M$ satisfies the $\kappa$-covering property for $V$.

(3) If $\kappa$ is regular, then $M$ also satisfies a slightly stronger property: for every ordinal $\alpha$ and every function $f: \alpha \rightarrow[M]^{<\kappa}$, there is a function $F \in M$ such that $\operatorname{dom}(F)=\alpha, f(\beta) \subseteq F(\beta)$, and $|F(\beta)|<\kappa$ for all $\beta<\alpha$.

The following theorem of Bukovský is very useful. It shows that a model of ZFC is a ground if and only if it has the uniform covering property.

Fact 3.9 (Bukovský [2]). Suppose $M \subseteq V$ is a transitive model of ZFC containing all ordinals. Let $\kappa$ be a regular uncountable cardinal. Then the following are equivalent:

(1) $M$ satisfies the $\kappa$-uniform covering property for $V$.

(2) There is a poset $\mathbb{P} \in M$ and an $(M, \mathbb{P})$-generic $G$ such that $\mathbb{P}$ satisfies the $\kappa$-c.c. in $M$, and $M[G]=V$.

\footnotetext{
${ }^{1}$ In Friedman-Fuchino-Sakai [3], this property is referred as " $M \kappa$-globally coves $V "$.
} 
See also Friedman-Fuchino-Sakai [3] and Schindler [13] for simple and modern proofs of Bukovský's theorem. Friedman-Fuchino-Sakai [3] gave a proof using an infinitary logic, and Schindler [13] used an extender algebra.

\section{The Covering AND the Approximation properties of Models of $\mathrm{ZFC}-\mathrm{P}$}

In this section, we discuss the covering and the approximation properties of submodels of $\mathcal{H}(\chi)$. These will be used in the next section.

First let us make the following note:

Note 4.1. We use the property that "every set can be coded by a set of ordinals". This is implied by $\mathrm{ZF}-\mathrm{P}+$ the well-ordering theorem, and, if $\chi$ is regular uncountable, then $\mathcal{H}(\chi)$ satisfies the well-ordering theorem of course. Natural submodels of $\mathcal{H}(\chi)$ satisfy only ZFC-P. However, it is known that ZFC-P does not imply the well-ordering theorem (Zarach [17]). So ZFC-P does not imply our required property. Moreover the collection schemes do not follow from ZFC-P (Zarach [18]). To avoid those difficulties, we identify the axiom of choice with the well-ordering theorem, and the replacement schemes with the collection schemes. Hence "a model of ZFC-P" means "a model of ZF-P+the collection schemes+the well-ordering theorem".

Here we explicitly define the covering and the approximation properties of submodels of $\mathcal{H}(\chi)$.

Definition 4.2. Let $\kappa<\chi$ be regular uncountable cardinals. Let $M \subseteq \mathcal{H}(\chi)$ be a transitive model of $\mathrm{ZFC}-\mathrm{P}$ with $\chi \subseteq M$.

(1) $M$ satisfies the $\kappa$-covering property for $\mathcal{H}(\chi)$ if for every set $A$ of ordinals with $|A|<\kappa$ and $A \subseteq \chi($ so $A \in \mathcal{H}(\chi))$, there is some set $B \in M$ of ordinals such that $|B|<\kappa$ and $A \subseteq B$.

(2) $M$ satisfies the $\kappa$-approximation property for $\mathcal{H}(\chi)$ if for every bounded subset $A \subseteq \chi($ so $A \in \mathcal{H}(\chi))$, if $A \cap x \in M$ for every $x \in M \cap[\chi]^{<\kappa}$, then $A \in M$.

(3) $M$ satisfies the $\kappa$-uniform covering property for $\mathcal{H}(\chi)$ if for every $\alpha<\chi$ and $f: \alpha \rightarrow \chi$ (so $f \in \mathcal{H}(\chi))$, there is $F \in M$ such that $\operatorname{dom}(F)=\alpha$, $f(\beta) \in F(\beta)$, and $|F(\beta)|<\kappa$ for all $\beta<\alpha$.

The following is a variation of Fact 3.4.

Lemma 4.3. Let $\kappa<\chi$ be regular uncountable cardinals with $\kappa^{+}<\chi$. Let $M, N \subseteq$ $\mathcal{H}(\chi)$ be transitive models of $Z F C-P$ with $\chi \subseteq M \cap N$. If $M$ and $N$ satisfy the $\kappa$-uniform covering and the $\kappa$-approximation properties for $\mathcal{H}(\chi)$, and $\mathcal{P}(\kappa) \cap M=$ $\mathcal{P}(\kappa) \cap N$, then $M=N$. 
Proof. We repeat the proof of Fact 3.4.

First note that $\left(\kappa^{+}\right)^{M}=\left(\kappa^{+}\right)^{N}=\kappa^{+}$; this is immediate from the $\kappa$-uniform covering property of $M$ and $N$. Since $\mathcal{P}(\kappa) \cap M=\mathcal{P}(\kappa) \cap N$, we have that $\mathcal{P}(\alpha) \cap M=\mathcal{P}(\alpha) \cap N$ for every $\alpha<\kappa^{+}$, which we can verify as follows: For $\alpha<\kappa^{+}$, we can take a set $X \subseteq \kappa \times \kappa$ such that $X \in M$ and $\langle\kappa, X\rangle$ is isomorphic to $\alpha$. There is a unique isomorphism $\pi$ from $\langle\kappa, X\rangle$ onto $\alpha$. Since $\mathcal{P}(\kappa) \cap M=\mathcal{P}(\kappa) \cap N$, we have $X \in N$, hence $\pi$ is also in $N$. Using this $\pi$, we can check that $\mathcal{P}(\alpha) \cap M=\mathcal{P}(\alpha) \cap N$. We also note that $M$ and $N$ satisfy the $\kappa$-covering property for $\mathcal{H}(\chi)$.

Since both $M$ and $N$ are models of ZFC-P, every set in $M$ and $N$ can be coded by a set of ordinals (see Note 4.1). Thus, in order to show $M=N$, it is sufficient to show that, for every set $A \subseteq \chi$, we have $A \in M \Longleftrightarrow A \in N$.

First we show that $[\chi]^{<\kappa} \cap M=[\chi]^{<\kappa} \cap N$. Fix $A \in[\chi]^{<\kappa} \cap M$. We see $A \in N$, and the converse follows from the same argument. By induction on $i<\kappa$, we take $\left\langle x_{i}, y_{i}: i<\kappa\right\rangle$ as follows:

(1) $x_{i} \in[\chi]^{<\kappa} \cap M$ and $y_{i} \in[\chi]^{<\kappa} \cap N$.

(2) $\left\langle x_{i}: i<\kappa\right\rangle$ and $\left\langle y_{i}: i<\kappa\right\rangle$ are $\subseteq$-increasing.

(3) $A \subseteq x_{i} \cup y_{i} \subseteq x_{i+1} \cap y_{i+1}$.

Suppose $\left\langle x_{j}, y_{j}: j<i\right\rangle$ is defined. Let $z=\bigcup_{j<i}\left(x_{j} \cup y_{j}\right)$. We have $z \in[\chi]^{<\kappa}$. By the $\kappa$-covering property of $M$ and $N$, we can find $x_{i} \in M \cap[\chi]^{<\kappa}$ and $y_{i} \in N \cap[\chi]^{<\kappa}$ with $z \subseteq x_{i}, y_{i}$.

Now let $B=\bigcup_{i<\kappa} x_{i}=\bigcup_{i<\kappa} y_{i}$. Next we check $B \in M \cap N$. To see $B \in M$, we use the $\kappa$-approximation property of $M$. Thus take $c \in M \cap[\chi]^{<\kappa}$. Since $|c|<\kappa$ and $B=\bigcup_{i<\kappa} x_{i}$, there must be $k<\kappa$ with $B \cap c \subseteq x_{k}$. Then $B \cap c=x_{k} \cap x \in M$. Hence $B \in M$ by the approximation property of $M$. We also know that $B \in N$ by the same argument.

Let $\delta=\operatorname{ot}(B)<\kappa^{+}$. Let $\pi: B \rightarrow \delta$ be the transitive collapse map. We know $\pi \in M \cap N$. Let $D=\pi^{\prime \prime} A \in M \cap \mathcal{P}(\delta)$. Since $\mathcal{P}(\delta) \cap M=\mathcal{P}(\delta) \cap N$, we have that $D \in N$. Then $A=\pi^{-1} " D \in N$.

Now we have $[\chi]^{<\kappa} \cap M=[\chi]^{<\kappa} \cap N$. Finally we show that for every set $A \subseteq \chi$, we have $A \in M \Longleftrightarrow A \in N$. Take a set $A \in M \cap \mathcal{P}(\chi)$. For every $x \in[\chi]^{<\kappa} \cap M$, we have that $A \cap x$ is in $M$. On the other hand, since $[\chi]^{<\kappa} \cap M=[\chi]^{<\kappa} \cap N$, we have that $A \cap x \in N$ for every $x \in[\chi]^{<\kappa}$. Then $A \in N$ by the $\kappa$-approximation property of $N$. The converse follows from the same argument.

By Facts 3.5 and 3.9, if $M$ and $\mathcal{H}(\chi)$ are models of ZFC, and $M$ is a class of $\mathcal{H}(\chi)$, then the uniform covering property of $M$ implies the approximation property using Bukovský's theorem. However we do not know that Fact 3.9 is valid for models of ZFC-P, so the implication is not clear for submodels of $\mathcal{H}(\chi)$. Here, we give a direct proof of the approximation property of submodels of $\mathcal{H}(\chi)$ from the uniform covering property. 
To do it, we make some observations about trees. For a tree $T$ of height $\alpha$ and $\beta<\alpha$, let $T_{\beta}$ be the $\beta$-th level of $T$.

The following is a well-known theorem of Kurepa:

Fact 4.4 (Kurepa [8]). Let $\kappa<\lambda$ be regular cardinals. For every tree $T$ of height $\lambda$, if $\left|T_{\alpha}\right|<\kappa$ for every $\alpha<\lambda$, then $T$ has a cofinal branch.

Lemma 4.5. Let $\kappa<\lambda$ be regular cardinals. Let $T$ be a tree of height $\lambda$. Suppose that $\left|T_{\alpha}\right|<\kappa$ for every $\alpha<\lambda$. Then $T$ has fewer than $\kappa$ many cofinal branches.

Proof. Suppose to the contrary that $T$ has $\kappa$ cofinal branches $\left\langle B_{i}: i<\kappa\right\rangle$. For each $i<j<\kappa$, there is some $\alpha(i, j)<\lambda$ such that $B_{i} \cap T_{\alpha(i, j)} \neq B_{j} \cap T_{\alpha(i, j)}$. Put $\alpha=\sup \{\alpha(i, j): i<j<\kappa\}$. We have $\alpha<\lambda$ since $\kappa<\lambda$. Then, for every $i<j<\kappa$, we know $B_{i} \cap T_{\alpha} \neq B_{j} \cap T_{\alpha}$. Thus $T_{\alpha}$ has cardinality at least $\kappa$, which is a contradiction.

Lemma 4.6. Let $\kappa$ be a regular cardinal, and $\mu>\kappa$ an ordinal with $\operatorname{cf}(\mu)>\kappa$. Let $T$ be a tree of height $\mu$. Suppose that $\left|T_{\alpha}\right|<\kappa$ for every $\alpha<\mu$. Let $W \subseteq V$ be a transitive model of ZFC containing all ordinals. If $T \in W$, then every cofinal branch of $T$ in $V$ belongs to $W$.

Proof. Case 1: $\operatorname{cf}(\mu)^{W}=\mu$. We work in $W$. By Lemma 4.5, we have that $T$ has fewer than $\kappa$ many cofinal branches. Let $\nu<\kappa$ and $\left\langle B_{i}: i<\nu\right\rangle$ be an enumeration of all cofinal branches of $T$.

In $V$, suppose to the contrary that there exists a cofinal branch $B$ of $T$ with $B \notin W$. For each $i<\nu$, there is $\alpha(i)<\mu$ such that $T_{\alpha(i)} \cap B \neq T_{\alpha(i)} \cap B_{i}$. Since $\operatorname{cf}(\mu)^{V}>\kappa$, we have that $\alpha=\sup \{\alpha(i)+1: i<\nu\}<\mu$. Pick $t \in B \cap T_{\alpha}$. We know $t \notin B_{i}$ for every $i<\nu$. On the other hand, by Kurepa's theorem (Fact 4.4) applied to $W$, there is a cofinal branch $B^{\prime} \in W$ with $t \in B^{\prime}$. Then $B^{\prime}=B_{i}$ for some $i<\nu$, but $t \in B_{i}$, which is a contradiction.

Case 2: $\operatorname{cf}(\mu)^{W}<\mu$. We work in $W$. Fix a cofinal set $X \subseteq \mu$ with order type $\operatorname{cf}(\mu)^{W}$, and consider the subtree $T^{*}=\bigcup_{\alpha \in X} T_{\alpha}$ of $T$. Then $T^{*}$ is a tree of height $\operatorname{cf}(\mu)^{W}$, and $\left|T_{\alpha}^{*}\right|<\kappa$ for every $\alpha<\operatorname{cf}(\mu)$. Moreover, if $B^{*} \subseteq T^{*}$ is a cofinal branch of $T^{*}$, then there is a unique cofinal branch $B \subseteq T$ with $B \cap T^{*}=B^{*}$. Now let $B \subseteq T$ be a cofinal branch of $T$ with $B \in V$. Let $B^{*}=B \cap T^{*} . B^{*}$ is a cofinal branch of $T^{*}$. By (1), we have that $B^{*} \in W$, and there is a cofinal branch $B^{\prime} \subseteq T$ with $B^{\prime} \cap T^{*}=B^{*}$. Then it is clear that $B^{\prime}=B$, hence $B \in W$.

Now we prove the main result of this section.

Lemma 4.7. Let $\theta$ be a strong limit cardinal, and $\kappa<\theta$ be a regular uncountable cardinal. Let $\chi=\theta^{+}$, and suppose there is a transitive model $W \subseteq V$ of $Z F C$ such that $M=\mathcal{H}(\chi)^{W}=\mathcal{H}(\chi) \cap W$. If $M$ satisfies the $\kappa$-uniform covering property for $\mathcal{H}(\chi)$, then $M$ satisfies the $\kappa^{+}$-approximation property for $\mathcal{H}(\chi)$. 
Proof. First note the following:

(1) For every $\alpha$ with $\kappa \leq \alpha<\chi, \alpha$ is regular in $M$ if and only if $\alpha$ is regular in $V$.

(2) In particular, if $\kappa \leq \alpha<\chi$, then $\alpha$ is a cardinal in $M$ if and only if $\alpha$ is a cardinal in $V$.

(3) The set $M \cap^{<\theta} 2$ is in $M$.

(1) and (2) follow from the $\kappa$-uniform covering property of $M$. For (3), since $\theta$ is strong limit, $\theta$ is also a strong limit cardinal in $W \subseteq V$. Thus $W \cap{ }^{<\theta} 2\left(=\left({ }^{<\theta} 2\right)^{W}\right)$ has cardinality $\theta$. Hence we have

$$
M \cap{ }^{<\theta} 2=W \cap{ }^{<\theta} 2 \in \mathcal{H}(\chi) \cap W=M .
$$

We prove the following by induction on $\alpha<\chi$ : For every $A \subseteq \alpha$, if $A \cap x \in M$ for every $x \in M \cap[\alpha]^{<\kappa^{+}}$, then $A \in M$.

Fix $\alpha<\chi, A \subseteq \alpha$, and suppose $A \cap x \in M$ for every $x \in M \cap[\alpha]^{<\kappa^{+}}$. By the induction hypothesis, we have that $A \cap \beta \in M$ for every $\beta<\alpha$.

It is clear in the case $\alpha<\kappa^{+}$. Thus suppose $\alpha \geq \kappa^{+}$.

Case 1: $\alpha$ is not a cardinal. By the above remark (2), we have that $\alpha$ is not a cardinal in $M$. Put $\lambda=|\alpha|^{M}$. Take a bijection $\pi: \lambda \rightarrow \alpha$ with $\pi \in M$, and let $B=\pi^{-1 "} A \subseteq \lambda$. It is easy to check that $B \cap x \in M$ for every $x \in[\lambda]^{<\kappa} \cap M$. By the induction hypothesis, we have $B \in M$. Then $A=\pi$ " $B \in M$.

Case 2: $\alpha$ is a cardinal with $\operatorname{cf}(\alpha)<\kappa^{+}$. Note that $\alpha \leq \theta$ by remark (2).

First we claim the following:

Claim 4.8. The set $M \cap[\alpha]^{<\kappa^{+}}$is stationary in $[\alpha]^{<\kappa^{+}}$

Proof. First note that we do not require that $M \cap[\alpha]^{<\kappa^{+}} \in M$.

To show the assertion, take a function $f:[\alpha]^{<\omega} \rightarrow \alpha$. We will find $x \in M \cap[\alpha]^{<\kappa^{+}}$ which is closed under $f$ and $\kappa \subseteq x$. There is a bijection $\pi \in M$ from $[\alpha]^{<\omega}$ onto $\alpha$. Hence, by the $\kappa$-uniform covering property of $M$, there is $F \in M$ such that $\operatorname{dom}(F)=[\alpha]^{<\omega}, f(s) \in F(s) \subseteq \alpha$, and $|F(s)|<\kappa$. Since $M$ is a model of ZFC-P, we can find $x \in M \cap[\alpha]^{<\kappa^{+}}$such that $\kappa \subseteq x$ and $F(s) \subseteq x$ for all $s \in[x]^{<\omega}$. Then $x$ is clearly closed under $f$.

$\square[$ Claim $]$

By remark (3), we have that $M \cap\{x \subseteq \alpha: x$ is bounded in $\alpha\} \in M$. Fix $\mu \in \chi$ and a one-to one enumeration $\left\langle B_{i}: i<\mu\right\rangle \in M$ of $M \cap\{x \subseteq \alpha: x$ is bounded in $\alpha$. Fix $X \subseteq \alpha$ such that $X \in M,|X|<\kappa^{+}$, and $X$ is unbounded in $\alpha$. We have that $A \cap \beta \in M$ for every $\beta<\alpha$. Thus, for each $\beta \in X$, there is a unique $i(\beta)<\mu$ with $A \cap \beta=B_{i(\beta)}$. We will see that the set $\{i(\beta): \beta \in X\}$ is in $M$, then $A=\bigcup\left\{B_{i(\beta)}: \beta \in X\right\} \in M$, as required. 
Since $M$ satisfies the $\kappa$-uniform covering property, $M$ satisfies the $\kappa^{+}$-covering property. Thus we can find $Y \in M \cap[\mu]^{<\kappa^{+}}$with $\{i(\beta): \beta \in X\} \subseteq Y$.

By the claim above, we can find $N \prec \mathcal{H}(\chi)$ such that $|N|<\kappa^{+}, \kappa \subseteq N$, $\sup (N \cap \alpha)=\alpha,\left\langle B_{i}: i<\mu\right\rangle, X, Y, A \in N$, and $N \cap \alpha \in M$. Note that $X, Y \subseteq N$ since $|X|,|Y|<\kappa^{+}$.

For every $\beta \in X$ and $i \in Y$, we have that $i=i(\beta) \Longleftrightarrow A \cap \beta=B_{i}$. By the elementarity of $N$ and $X, Y \subseteq N$, for every $\beta \in X$ and $i \in Y$, we know that $i=i(\beta) \Longleftrightarrow A \cap \beta \cap N=B_{i} \cap(N \cap \alpha)$.

Since $|N|<\kappa^{+}$and $N \cap \alpha \in M$, we have $N \cap \alpha \in M \cap[\alpha]^{<\kappa^{+}}$, hence $A \cap N=$ $A \cap(N \cap \alpha) \in M$. Thus, in $M$, for each $\beta \in X, i(\beta)$ is definable as the unique $i \in Y$ with $(A \cap N) \cap \beta=B_{i} \cap(N \cap \alpha)$. Hence we have $\langle i(\beta): \beta \in X\rangle \in M$, and $A=\bigcup_{\beta \in X} B_{i(\beta)} \in M$.

Case 3: $\alpha$ is a cardinal with $\operatorname{cf}(\alpha) \geq \kappa^{+}$. As in Case 2, we have that $\alpha \leq \theta$.

Let $f: \alpha \rightarrow 2$ be the characteristic function of $A$. It is enough to see that $f \in M$. Since $A \cap \beta \in M$ for every $\beta<\alpha$, we have that $f \uparrow \beta \in M$ for every $\beta<\alpha$. Since $\alpha \leq \theta$, the set ${ }^{<\alpha} 2 \cap M$ is in $M$ by remark (3). Fix $\mu<\chi$ and a one-to-one enumeration $\left\langle g_{i}: i<\mu\right\rangle \in M$ of $<\alpha 2 \cap M$. Define $h: \alpha \rightarrow \mu$ by $h(\beta)=i \Longleftrightarrow f \uparrow \beta=g_{i}$. By the $\kappa$-uniform covering property of $M$, there is $H \in M$ with $h(\beta) \in H(\beta)$ and $|H(\beta)|<\kappa$. We may assume that for every $i \in H(\beta), \operatorname{dom}\left(g_{i}\right)=\beta$. Put $T^{\prime}=\left\{g_{i}: i \in H(\beta), \beta<\alpha\right\} \in M$. We know that $f \uparrow \beta \in T^{\prime}$ for all $\beta<\alpha$.

In $M$, let $T$ be the set of all $g \in T^{\prime}$ such that $g\left\lceil\gamma \in T^{\prime}\right.$ for every $\gamma<\operatorname{dom}(g)$. Again, we have $f\lceil\beta \in T$ for every $\beta<\alpha$. Thus $T$ is a tree of height $\alpha$ and for every $g \in T$ and $\gamma<\operatorname{dom}(g)$, we have $g\lceil\gamma \in T$. The $\beta$-th level of $T$ is a subset of $\left\{g_{i}: i \in H(\beta)\right\}$. Since $|H(\beta)|<\kappa$, we have that $\left|T_{\beta}\right|<\kappa$ for every $\beta<\alpha$. The set $B=\{f \uparrow \beta: \beta<\alpha\}$ is a cofinal branch of $T$. $T$ is a tree in a transitive model $W$ of ZFC. Applying Lemma 4.6 to $W$ and $T$, we have $B \in W$. Then $B \in M$ since $M=\mathcal{H}(\chi)^{W}$, hence $f \in M$.

We have the following as an immediate corollary of Lemma 4.7.

Corollary 4.9. Let $W \subseteq V$ be a transitive model of $Z F C$ containing all ordinals. Let $\kappa$ be a regular uncountable cardinal. If $W$ satisfies the $\kappa$-uniform covering property for $V$, then $W$ satisfies the $\kappa^{+}$-approximation property for $V$, and $W$ is definable in $V$ with some parameters.

Proof. For each strong limit cardinal $\theta>\kappa$, we have that $\mathcal{H}\left(\theta^{+}\right)^{W}$ is of the form $W \cap \mathcal{H}\left(\theta^{+}\right)$and satisfies the $\kappa^{+}$-approximation property for $\mathcal{H}\left(\theta^{+}\right)$by Lemma 4.7. Then it is clear that $W$ satisfies the $\kappa^{+}$-approximation property for $V$.

Let $r=\mathcal{P}\left(\kappa^{+}\right) \cap W$. Now $W$ is definable in $V$ with parameters $r$ and $\kappa^{+}$as follows: $x \in W$ if and only if there is a strong limit cardinal $\theta>\kappa$ and the unique 
transitive model $M \subseteq \mathcal{H}\left(\theta^{+}\right)$of $\mathrm{ZFC}-\mathrm{P}$ such that $\theta^{+} \subseteq M, M \cap \mathcal{P}\left(\kappa^{+}\right)=r, M$ satisfies the $\kappa^{+}$-uniform covering and the $\kappa^{+}$-approximation properties for $\mathcal{H}\left(\theta^{+}\right)$, and $x \in M$.

Note 4.10. (1) If $W \subseteq V$ satisfies the uniform covering property for $V$, then $W$ is a ground by Bukovsky's theorem, hence $W$ is definable in $V$ by Laver and Woodin's result. In this sense, the definablity of $W$ satisfying the uniform covering property is not a new result.

(2) On the other hand, in the proof of Corollary 4.9, it is not necessary that $V$ satisfies the replacement scheme for the formulas of the language $\{\in, W\}$. So Corollary 4.9 shows that every $W \subseteq V$ satisfying the uniform covering property is definable, regardless of the replacement scheme for the formulas of the language $\{\in, W\}$.

(3) The $\kappa^{+}$-approximation property in the previous corollary cannot be strengthened to the $\kappa$-approximation property; suppose there exists a $\kappa$-Suslin tree $T$, and let $\mathbb{P}$ be a $\kappa$-c.c. poset adding a cofinal branch of $T$. If $G$ is $(V, \mathbb{P})$ generic, then $V$ satisfies the $\kappa$-uniform covering property for $V[G]$, but a cofinal branch of $T$ witnesses that $V$ does not satisfy the $\kappa$-approximation property for $V[G]$.

(4) On the other hand, if $W \subseteq V$ satisfies the $\kappa$-uniform covering property for $V$ (hence $V$ is a $\kappa$-c.c. forcing extension of $W$ ) and there is no $\kappa$-Suslin tree in $W$, then $W$ satisfies the $\kappa$-approximation property for $V$ (see Usuba $[15])$.

\section{The STRONG DDG}

In this section, we prove that the strong DDG is a theorem of ZFC.

Proposition 5.1. The strong DDG holds.

Proof. Fix a set $X$. We will construct a ground $W$ of $V$ such that $W \subseteq W_{r}$ for all $r \in X$ simultaneously as follows. Then $W$ is a ground of each $W_{r}$ by Fact 3.1.

For each $r \in X$, there is a poset $\mathbb{P}_{r} \in W_{r}$ and a $\left(W_{r}, \mathbb{P}_{r}\right)$-generic $G_{r}$ with $V=W_{r}\left[G_{r}\right]$. Fix a regular uncountable $\kappa$ such that $|X|<\kappa$ and $\left|\mathbb{P}_{r}\right|<\kappa$ for every $r \in X$. Then by Facts 3.5 and 3.9, each $W_{r}$ satisfies the $\kappa$-uniform covering and the $\kappa$-approximation properties for $V$.

Fix a strong limit cardinal $\theta>\kappa$, and let $\chi=\theta^{+}$. First we show that there exists a transitive model $M$ of ZFC-P such that $\chi \subseteq M \subseteq \mathcal{H}(\chi)$ and $M$ satisfies the $\kappa^{++}$-uniform covering and the $\kappa^{++}$-approximation properties for $\mathcal{H}(\chi)$.

Let $\gamma=\chi^{<\chi}$, and $\left\langle f_{\xi}: \xi<\gamma\right\rangle$ be an enumeration of $<\chi \chi$. Now define $h: \chi \times \gamma \rightarrow$ $\chi$ as follows: $h(\alpha, \xi)=f_{\xi}(\alpha)$ if $\alpha \in \operatorname{dom}\left(f_{\xi}\right)$, and $h(\alpha, \xi)=0$ otherwise.

Claim 5.2. There is a function $H$ such that $\operatorname{dom}(H)=\chi \times \gamma, h(\alpha, \xi) \in H(\alpha, \xi) \subseteq$ $\chi,|H(\alpha, \xi)|<\kappa^{+}$for $\alpha<\chi, \xi<\gamma$, and $H \in \bigcap_{r \in X} W_{r}$. 
Proof. By induction on $i<\kappa$, we define $H_{i, r}$ for $r \in X$ as follows:

(1) $H_{i, r}$ is a function with $H_{i, r} \in W_{r}$.

(2) $\operatorname{dom}\left(H_{i, r}\right)=\chi \times \gamma, h(\alpha, \xi) \in H_{i, r}(\alpha, \xi) \subseteq \chi$, and $\left|H_{i, r}(\alpha, \xi)\right|<\kappa$ for all $\alpha<\chi$ and $\xi<\gamma$.

(3) For all $\alpha<\chi$ and $\xi<\gamma, \bigcup_{j<i, s \in X}, H_{j, s}(\alpha, \xi) \subseteq H_{i, r}(\alpha, \xi)$.

Let $i<\kappa$ and suppose $H_{j, s}$ was defined for all $s \in X$ and $j<i$. Fix $r \in X$. To define $H_{i, r}$. let $H^{\prime}$ be such that $H^{\prime}(\alpha, \xi)=\bigcup_{j<i, s \in X} H_{j, s}(\alpha, \xi)$ for all $\alpha<\chi$ and $\xi<\gamma$. Since $|X|$ and $i$ are less than $\kappa$, we have that $\left|H^{\prime}(\alpha, \xi)\right|<\kappa$ for all $\alpha<\chi$ and $\xi<\gamma . W_{r}$ satisfies the $\kappa$-uniform covering property for $V$, hence we can find $H_{i, r} \in W_{r}$ such that $H^{\prime}(\alpha, \xi) \subseteq H_{i, r}(\alpha, \xi) \subseteq \chi$ and $\left|H_{i, r}(\alpha, \xi)\right|<\kappa$ for all $\alpha<\chi$ and $\xi<\gamma$. It is clear that $h(\alpha, \xi) \in H_{i, r}(\alpha, \xi)$.

Finally, let $H$ be such that $H(\alpha, \xi)=\bigcup_{i<\kappa} H_{i, r}(\alpha, \xi)$ for some (in fact all) $r \in X$. Clearly $h(\alpha, \xi) \in H(\alpha, \xi)$ and $|H(\alpha, \xi)|<\kappa^{+}$. We have to show that $H \in W_{r}$ for all $r \in X$.

Fix $r \in X$. Let $E=\{\langle\alpha, \xi, \eta\rangle \in \chi \times \gamma \times \chi: \eta \in H(\alpha, \xi)\}$. It is sufficient to show that $E \in W_{r}$. To show that $E \in W_{r}$, we use the $\kappa$-approximation property of $W_{r}$. So take $a \in[\chi \times \gamma \times \chi]^{<\kappa} \cap W_{r}$. We will see that $a \cap E \in W_{r}$. Let $d=\{\langle\alpha, \xi\rangle \in \chi \times \gamma: \exists \eta(\langle\alpha, \xi, \eta\rangle \in a \cap E)\}$. We have $|d|<\kappa$. For each $\langle\alpha, \xi\rangle \in d$, the set $\{\eta<\chi:\langle\alpha, \xi, \eta\rangle \in a \cap E\} \subseteq H(\alpha, \xi)$ has cardinality $<\kappa$, thus we can find $i(\alpha, \xi)<\kappa$ with $\{\eta<\chi:\langle\alpha, \xi, \eta\rangle \in a \cap E\} \subseteq H_{i(\alpha, \xi), r}(\alpha, \xi)$. Put $i^{*}=\sup \{i(\alpha, \xi):\langle\alpha, \xi\rangle \in d\}<\kappa$. We have $\{\eta<\chi:\langle\alpha, \xi, \eta\rangle \in a \cap E\} \subseteq H_{i^{*}, r}(\alpha, \xi)$ for all $\langle\alpha, \xi\rangle \in d$. Then $a \cap E=\left\{\langle\alpha, \xi, \eta\rangle \in a: \eta \in H_{i^{*}, r}(\alpha, \xi)\right\} \in W_{r} . \quad \square[$ Claim]

Fix a bijection $\pi: \chi \times \gamma \times \chi \rightarrow \gamma$ with $\pi \in L$, and let $A=\pi$ " $H$. Clearly $A \in W_{r}$ for all $r \in X$. Since $A$ is a set of ordinals, we have that $L[A]$ is a model of $\mathrm{ZFC}$, and $H \in L[A]$. Now let $M=\mathcal{H}(\chi)^{L[A]}$. We know $M=L[A] \cap \mathcal{H}(\chi)$ and $\chi \subseteq M \subseteq \mathcal{H}(\chi)$, and since $A \in W_{r}$ for all $r \in X$, we have $M \subseteq \bigcap_{r \in X} W_{r}$.

Claim 5.3. $M$ satisfies the $\kappa^{+}$-uniform covering property for $\mathcal{H}(\chi)$.

Proof. Take $f: \alpha \rightarrow \chi$ for some $\alpha<\chi$. Since $\left\langle f_{\xi}: \xi<\gamma\right\rangle$ is an enumeration of $<\chi \chi$, there is some $\xi^{*}<\gamma$ with $f=f_{\xi^{*}}$. Now define the function $F$ such that $\operatorname{dom}(F)=\alpha$ and $F(\beta)=H\left(\beta, \xi^{*}\right)$. By the choice of $H$, we have that $f(\beta)=$ $f_{\xi^{*}}(\beta) \in H\left(\beta, \xi^{*}\right)=F(\beta)$ and $|F(\beta)|<\kappa^{+}$. Moreover, since $H \in L[A]$, we have $F \in L[A]$, and $F \in \mathcal{H}(\chi)^{L[A]}=M$.

$\square$ [Claim]

$M$ is $\mathcal{H}(\chi) \cap L[A]$ and $\chi$ is the successor of the strong limit cardinal $\theta$. So $M$ satisfies the $\kappa^{++}$-approximation property for $\mathcal{H}(\chi)$ by Lemma 4.7.

In summary, for each strong limit cardinal $\theta>\kappa$, we can find a transitive model $M$ of ZFC-P such that $\theta^{+} \subseteq M \subseteq \mathcal{H}\left(\theta^{+}\right), M \subseteq \bigcap_{r \in} W_{r}$, and $M$ satisfies the $\kappa^{++}$ uniform covering and the $\kappa^{++}$-approximation properties for $\mathcal{H}\left(\theta^{+}\right)$. Note that, by Lemma 4.3 (and Note 4.1), letting $r=\mathcal{P}\left(\kappa^{++}\right) \cap M, M$ is a unique transitive model 
$N$ of ZFC-P such that $\theta^{+} \subseteq N \subseteq \mathcal{H}\left(\theta^{+}\right), r=\mathcal{P}\left(\kappa^{++}\right) \cap N$, and $N$ satisfies the $\kappa^{++}$-uniform covering and the $\kappa^{++}$-approximation properties for $\mathcal{H}\left(\theta^{+}\right)$.

For each $p \subseteq \mathcal{P}\left(\kappa^{++}\right)$, let $I_{p}$ be the class of all strong limit cardinals $\theta>\kappa$ such that there exists a transitive model $M$ of ZFC-P such that $\theta^{+} \subseteq M \subseteq \mathcal{H}\left(\theta^{+}\right)$, $p=M \cap \mathcal{P}\left(\kappa^{++}\right), M \subseteq \bigcap_{r \in} W_{r}$, and $M$ satisfies the $\kappa^{++}$-uniform covering and the $\kappa^{++}$-approximation properties for $\mathcal{H}\left(\theta^{+}\right)$. By the pigeonhole argument, there must be one set $p^{*} \subseteq \mathcal{P}\left(\kappa^{++}\right)$such that $I_{p^{*}}$ forms a proper class. For each $\theta \in I_{p^{*}}$, fix a unique transitive model $M^{\theta}$ of ZFC-P such that $\theta^{+} \subseteq M^{\theta} \subseteq \mathcal{H}\left(\theta^{+}\right), M^{\theta} \subseteq$ $\bigcap_{r \in X} W_{r}, p^{*}=\mathcal{P}\left(\kappa^{++}\right) \cap M^{\theta}$, and $M^{\theta}$ satisfies the $\kappa^{++}$-uniform covering and the $\kappa^{++}$-approximation properties for $\mathcal{H}\left(\theta^{+}\right)$. The sequence $\left\langle M^{\theta}: \theta \in I_{p^{*}}\right\rangle$ is coherent: For $\theta<\theta^{\prime}$ from $I_{p^{*}}$, we have $M^{\theta}=M^{\theta^{\prime}} \cap \mathcal{H}\left(\theta^{+}\right)$. This can be verified as follows: Since $M^{\theta^{\prime}} \cap \mathcal{H}\left(\theta^{+}\right)=\left\{x \in M^{\theta^{\prime}}: M^{\theta^{\prime}} \vDash "|\operatorname{trcl}(x)|<\theta^{+} "\right\} \in M^{\theta^{\prime}}$, it is easy to check that $M^{\theta^{\prime}} \cap \mathcal{H}\left(\theta^{+}\right)$is a transitive model of ZFC-P and satisfies the $\kappa^{++}$-uniform covering property for $\mathcal{H}\left(\theta^{+}\right)$. Then $M^{\theta}=M^{\theta^{\prime}} \cap \mathcal{H}\left(\theta^{+}\right)$by Lemma 4.3.

Finally let $W=\bigcup\left\{M^{\theta}: \theta \in I_{p^{*}}\right\}$. Note that $W$ is definable in $V$.

Claim 5.4. $W$ is a transitive model of ZFC containing all ordinals.

Proof. Transitivity is clear, and it is also clear that $W$ contains all ordinals. To show that $W$ is a model of ZFC, by Fact 2.2, it is enough to see that $N$ is closed under the Gödel operations, $N$ is almost universal, and $N$ satisfies the axiom of choice.

For every $\theta \in I_{p^{*}}, M^{\theta}$ is closed under the Gödel operations. Since $W=\bigcup\left\{M^{\theta}\right.$ : $\left.\theta \in I_{p^{*}}\right\}$ and $\left\langle M^{\theta}: \theta \in I_{p^{*}}\right\rangle$ is coherent, we have that $W$ is closed under the Gödel operations. The almost universality of $W$ is immediate from the coherency of $\left\langle M^{\theta}: \theta \in I_{p^{*}}\right\rangle$.

We know that $W$ is a model of ZF. For the axiom of choice in $W$, for each $x \in W$, there is $\theta$ with $x \in M^{\theta}$. $M^{\theta}$ is a model of ZFC-P, thus $M^{\theta}$ has a well-ordering on $x$. This well-ordering belongs to $W$, hence $M$ satisfies the axiom of choice as well.

$\square[$ Claim $]$

We know that $W$ is a transitive model of ZFC containing all ordinals. Clearly $W \subseteq \bigcap_{r \in X} W_{r}$. Moreover, $W$ satisfies the $\kappa^{++}$-uniform covering property for $V$. Then $W$ is a ground of $V$ by Fact 3.9. This completes the proof.

We know that the strong DDG is a theorem of ZFC. So the following fundamental properties become theorems of ZFC as well. Recall that a bedrock is a minimal ground, and a solid bedrock is a minimum ground ([12], [4]).

Corollary 5.5. (1) $\mathbb{M}$ is a transitive model of $Z F C$ and $g \mathbb{M}=\mathbb{M}$.

(2) $\mathbb{M}$ is a forcing invariant class, that is, for every forcing extension $V[G]$ of $V$, we have $\mathbb{M}=\mathbb{M}^{V[G]}$.

(3) The following are equivalent: 
(a) Every forcing extension of $V$ has only set many grounds.

(b) $V$ has only set many grounds.

(c) $\mathbb{M}$ is a solid bedrock of all forcing extensions of $V$.

(d) $\mathbb{M}$ is a solid bedrock of $V$.

(e) $\mathbb{M}$ is a ground of $V$.

(f) $V$ has a bedrock.

Proof. (1). In Fuchs-Hamkins-Reitz [4], it was shown that if the strong DDG holds then $\mathbb{M}$ is a model of ZFC (Theorem 22 in [4]). Now we proved the strong DDG is a theorem of ZFC, so $\mathbb{M}$ is a model of ZFC. By Corollary 51 in [4], we know that, if the DDG holds in all forcing extensions of $V$, then $g \mathbb{M}$ is the same as $\mathbb{M}$. Again, since the strong DDG is a theorem of ZFC, the DDG holds in all forcing extensions of $V$. Hence we have that $g \mathbb{M}=\mathbb{M}$.

(2) follows from (1) and Fact 2.11.

(3). (a) $\Rightarrow$ (b) is trivial.

(b) $\Rightarrow$ (c). If $V$ has only set many grounds, then by the strong DDG, $\mathbb{M}$ is a ground of $V$, hence is a solid bedrock of $V$. By (1), for every forcing extension $V[G]$, we have $\mathbb{M}^{V}=g \mathbb{M} \subseteq V \subseteq V[G]$, hence $\mathbb{M}^{V}=g \mathbb{M}$ is a ground of $V[G]$, and, by the definition of $g \mathbb{M}, \mathbb{M}^{V}$ is a solid bedrock of $V[G]$.

(c) $\Rightarrow(\mathrm{d}) \Rightarrow(\mathrm{e}) \Rightarrow(\mathrm{f})$ are also trivial.

(f) $\Rightarrow$ (e). Suppose $W \subseteq V$ is a bedrock. If $\mathbb{M} \subsetneq W$, then there is a ground $W^{\prime}$ and $x \in W$ with $x \notin W^{\prime}$. By the DDG, $W$ and $W^{\prime}$ have a common ground $\bar{W}$. We know $W=\bar{W}$ by the minimality of $W$, so $x \notin W$. This is a contradiction. Hence we have $\mathbb{M}=W$ is a ground of $V$.

(e) $\Rightarrow$ (c). We see that $\mathbb{M}$ is a solid bedrock of all forcing extensions $V[G]$ of $V$. Since $\mathbb{M}^{V}$ is a ground of $V$ and $V$ is a ground of $V[G]$, we have that $\mathbb{M}^{V}$ is a ground of $V[G]$. If $W \subseteq V[G]$ is a ground of $V[G]$, then by the DDG in $V[G], \mathbb{M}$ and $W$ have a common ground $\bar{W} \cdot \bar{W} \subseteq \mathbb{M} \subseteq V$, hence $\bar{W}$ is a ground of $V$, so $\mathbb{M} \subseteq W$.

$(\mathrm{c}) \Rightarrow(\mathrm{a})$. For counting the grounds of $V[G]$, we can take a poset $\mathbb{Q} \in \mathbb{M}$ and an $(\mathbb{M}, \mathbb{Q})$-generic $H$ such that $V=\mathbb{M}[H]$. Since $\mathbb{M}$ is a solid bedrock of $V[G]$, $\mathbb{M} \subseteq W \subseteq V[G]$ for every ground $W$ of $V[G]$. Hence by Fact 3.1, every ground $W$ of $V[G]$ is of the form $W\left[H^{\prime}\right]$ for some $H^{\prime} \subseteq \mathbb{Q}^{\prime} \subseteq \operatorname{ro}(\mathbb{Q})^{\mathbb{M}}$. This shows that $V[G]$ has only $\left(2^{\left|r o(\mathbb{Q})^{\mathbb{M}}\right|}\right)^{V[G]}$ many grounds.

The Ground Axiom is the assertion that $V$ has no proper ground (Reitz [12]). If $\mathbb{M}$ is a ground of $V$, then it is clear that $\mathbb{M}$ has no proper ground. Hence, if $\mathbb{M}$ is a ground of $V$, then $\mathbb{M}$ is a natural model of $\mathrm{ZFC}+$ the Ground Axiom. 


\section{HyPER-HUGE CARDinAlS}

In this section, we prove that if a hyper-huge cardinal exists, then $V$ has only set many grounds, so $\mathbb{M}$ is a ground of $V$. To do this, we prove the following proposition which is interesting in its own right.

Proposition 6.1. Suppose $\kappa$ is hyper-huge. Let $W$ be a ground of $V$. Then there is a poset $\mathbb{P} \in W$ and $a(W, \mathbb{P})$-generic $G$ such that $|\mathbb{P}|<\kappa$ and $V=W[G]$.

Proof. Fix a poset $\mathbb{Q} \in W$ and a $(W, \mathbb{Q})$-generic $H$ such that $V=W[H]$.

Fix an arbitrary inaccessible cardinal $\lambda>\kappa$ with $\mathbb{Q}, H \in V_{\lambda}$. Since $\kappa$ is hyperhuge, we can find an elementary embedding $j: V \rightarrow N$ such that the critical point $\kappa, \lambda<j(\kappa)$, and $N$ is closed under $j(\lambda)$-sequences. Let $j(W)=\bigcup_{\alpha} j\left(W_{\alpha}\right) . j(W)$ is a transitive model of ZFC. Note that $j(W)_{j(\alpha)}=j\left(W_{\alpha}\right)$ for every $\alpha$.

First we show that

$$
W_{j(\lambda)} \subseteq j\left(W_{\lambda}\right) \subseteq N_{j(\lambda)}=V_{j(\lambda)} .
$$

The equality $N_{j(\lambda)}=V_{j(\lambda)}$ follows from the closure property of $N$, and the inclusion $j\left(W_{\lambda}\right) \subseteq N_{j(\lambda)}$ follows from the elementarity of $j$. So the problem is the inclusion $W_{j(\lambda)} \subseteq j\left(W_{\lambda}\right)$. Since $j(\lambda)$ is inaccessible, both $W_{j(\lambda)}$ and $j\left(W_{\lambda}\right)$ are models of ZFC. Thus it is enough to check that if $x \in W_{j(\lambda)}$ is a set of ordinals, then $x \in j\left(W_{\lambda}\right)$.

We use a well-known argument using disjoint stationary subsets, which is due to Solovay. In $W$, fix a pairwise disjoint sequence $\left\langle S_{\alpha}: \alpha<j(\lambda)\right\rangle$ such that $S_{\alpha} \subseteq$ $j(\lambda) \cap \operatorname{cof}(\omega)^{W}$ and $S_{\alpha}$ is stationary in $j(\lambda)$ in $W$. Since $V=W[G]$ and $|\mathbb{Q}|<$ $\lambda<j(\lambda)$, each $S_{\alpha}$ is still stationary in $j(\lambda)$ in $V$. Let $j\left(\left\langle S_{\alpha}: \alpha<j(\lambda)\right\rangle\right)=$ $\left\langle S_{\alpha}^{*}: \alpha<j(j(\lambda))\right\rangle$.

Claim 6.2. For every $\alpha<\sup (j " j(\lambda)), \alpha \in j$ “ $j(\lambda)$ if and only if $S_{\alpha}^{*} \cap \sup (j$ “ $j(\lambda))$ is stationary in $\sup (j " j(\lambda))$ in $j(W)$.

Proof of Claim. First suppose $\alpha \in j$ " $j(\lambda)$. Take $\beta<j(\lambda)$ with $\alpha=j(\beta)$. We know $S_{\alpha}^{*}=j\left(S_{\beta}\right)$. We check that $S_{\alpha}^{*} \cap \sup \left(j^{\prime \prime} j(\lambda)\right)$ is stationary in $\sup \left(j^{\prime \prime} j(\lambda)\right)$ in $j(W)$. To do this, take a club $C \subseteq \sup (j " j(\lambda))$ with $C \in j(W)$. Because the critical point of $j$ is uncountable, we know that $j^{\prime \prime} j(\lambda)$ is an $\omega$-club in $\sup (j " j(\lambda))$ in $V$, that is, it is unbounded in $\sup (j " j(\lambda))$ and, for every $\xi \in \lim (\sup (j " j(\lambda)))$, if $\operatorname{cf}(\xi)=\omega$ in $V$ then $\xi \in j$ " $j(\lambda)$. Hence we know that $C \cap j$ " $j(\lambda)$ is an $\omega$-club in $V$. Put $D=j^{-1}$ " $(C \cap j " j(\lambda))$. Again, since the critical point of $j$ is uncountable, $D$ is an $\omega$-club in $j(\lambda) . S_{\beta}$ is stationary in $j(\lambda)$ in $V$ and $\operatorname{cf}(\xi)=\omega$ for every $\xi \in S_{\beta}$, so we have that $S_{\beta} \cap D \neq \emptyset$. Fix $\xi \in S_{\beta} \cap D$. Then $j(\xi) \in j\left(S_{\beta}\right) \cap C$, so $j(\xi) \in S_{\alpha}^{*} \cap C$.

For the converse, suppose $S_{\alpha}^{*} \cap \sup \left(j^{\prime} j(\lambda)\right)$ is stationary in $j(W)$. By the elementarity of $j, N$ is a forcing extension of $j(W)$ via poset $j(\mathbb{Q})$. We know $|j(\mathbb{Q})|<j(\lambda)$. Hence, if $E \in j(W)$ is stationary in $j(\lambda)$ in $j(W)$, then $E$ remains stationary in $j(\lambda)$ in $N$. This means that $S_{\alpha}^{*} \cap \sup \left(j^{\prime \prime} j(\lambda)\right)$ remains stationary in $N$. $\operatorname{cf}(\xi)=\omega$ for every $\xi \in S_{\alpha}^{*}$ and $j^{\prime \prime} j(\lambda)$ is an $\omega$-club, hence we have $S_{\alpha}^{*} \cap j$ " $j(\lambda) \neq \emptyset$. Take 
$j(\xi) \in S_{\alpha}^{*} \cap j^{\text {" }} j(\lambda)$. By the elementarity of $j$, there is some $\beta<\lambda$ such that $\xi \in S_{\beta}$. Hence $j(\xi) \in S_{j(\beta)}^{*} \cap S_{\alpha}^{*}$, and then $j(\beta)=\alpha$ because the sequence $\left\langle S_{\gamma}^{*}: \gamma<j(j(\lambda))\right\rangle$ is pairwise disjoint.

$\square[$ Claim $]$

By the claim, we have that

$j$ “ $j(\lambda)=\left\{\alpha<\sup (j “ j(\lambda)): S_{\alpha}^{*} \cap \sup (j " j(\lambda))\right.$ is stationary in $\sup (j " j(\lambda))$ in $\left.j(W)\right\}$.

Thus we have that $j " j(\lambda)$ is definable in $j(W)$, and $j " j(\lambda) \in j(W)$. Now we see that, if $x \in W_{j(\lambda)}$ is a set of ordinals, then $x \in j\left(W_{\lambda}\right)$. Clearly $j(x) \in j(W)$. Since $x \subseteq j(\lambda)$, we have that $j$ " $x=j(x) \cap j$ " $j(\lambda) \in j(W)$. Because $j$ " $j(\lambda) \in j(W)$, we have that $j\left\lceil j(\lambda) \in j(W)\right.$, thus $x=j^{-1}$ " $(j " x) \in j(W)$. Again, since $x \subseteq j(\lambda)$, we have $x \in j(W)_{j(\lambda)}=j\left(W_{\lambda}\right)$.

We know $W_{j(\lambda)} \subseteq j\left(W_{\lambda}\right) \subseteq N_{j(\lambda)}=V_{j(\lambda)} . V=W[H]$, hence $V_{j(\lambda)}=W_{j(\lambda)}[H]$, and $W_{j(\lambda)} \subseteq j\left(W_{\lambda}\right)=N_{j(\lambda)}=V_{j(\lambda)}=W_{j(\lambda)}[H]$. By Fact 3.1, there is a poset $\mathbb{Q}^{\prime} \subseteq$ $\operatorname{ro}(\mathbb{Q})$ and a $\left(j\left(W_{\lambda}\right), \mathbb{Q}^{\prime}\right)$-generic $H^{\prime}$ such that $j\left(W_{\lambda}\right)\left[H^{\prime}\right]=V_{j(\lambda)}=N_{j(\lambda)}$. Since $|\mathbb{Q}|<\lambda<j(\kappa)$, we have that $\left|\mathbb{Q}^{\prime}\right|<j(\kappa)$. We may assume that $\mathbb{Q}^{\prime} \in j\left(W_{\lambda}\right)_{j(\kappa)}$ $\left(=j\left(W_{\kappa}\right)\right)$. Therefore, $N$ satisfies the following statement:

There exists a poset $\mathbb{Q}^{\prime}$ and a $\left(j\left(W_{\lambda}\right), \mathbb{Q}^{\prime}\right)$-generic $H$ such that $\mathbb{Q}^{\prime} \in$ $j\left(W_{\kappa}\right)$ and $j\left(W_{\lambda}\right)\left[H^{\prime}\right]=N_{j(\lambda)}$.

By the elementarity of $j$, the following holds in $V$ :

There exists a poset $\mathbb{Q}^{\prime}$ and a $\left(W_{\lambda}, \mathbb{P}\right)$-generic $H^{\prime}$ such that $\mathbb{Q}^{\prime} \in W_{\kappa}$ and $W_{\lambda}\left[H^{\prime}\right]=V_{\lambda}$.

Thus, for every sufficiently large inaccessible cardinal $\lambda$, there exists a poset $\mathbb{P}$ and a $\left(W_{\lambda}, \mathbb{P}\right)$-generic $G$ such that $\mathbb{P} \in W_{\kappa}$ and $W_{\lambda}[G]=V_{\lambda}$. Since $\kappa$ is hyper-huge, there are proper class many inaccessible cardinals. Hence there must be a poset $\mathbb{P} \in V_{\kappa}$ and a filter $G \subseteq \mathbb{P}$ such that the collection

$\left\{\lambda: \lambda\right.$ is inaccessible, $G$ is $\left(W_{\lambda}, \mathbb{P}\right)$-generic, and $\left.W_{\lambda}[G]=V_{\lambda}\right\}$

forms a proper class. Then it is clear that $G$ is $(W, \mathbb{P})$-generic and $W[G]=V$.

Now the following is immediate from Proposition 6.1:

Corollary 6.3. If $\kappa$ is hyper-huge, then $V$ has only set many grounds.

Proof. The assertion follows from Fact 3.5 and Proposition 6.1. For each ground $W$ of $V$, one can find a poset $\mathbb{P}_{W} \in V_{\kappa}$ and a $(W, \mathbb{P})$-generic $G_{W}$ such that $W\left[G_{W}\right]=V$. By Lemma 3.6, for two grounds $W$ and $W^{\prime}$, if $\mathbb{P}_{W}=\mathbb{P}_{W^{\prime}}, \lambda=$ $\left|\mathbb{P}_{W}\right|^{W}=\left|\mathbb{P}_{W^{\prime}}\right|^{W^{\prime}}$, and $\mathcal{P}(\lambda)^{W}=\mathcal{P}(\lambda)^{W^{\prime}}$ then $W=W^{\prime}$. Thus the assignment $W \mapsto\left\langle\mathbb{P}_{W},|\mathbb{P}|^{W}, \mathcal{P}\left(|\mathbb{P}|^{W}\right)^{W}\right\rangle$ is an injection from the grounds to $V_{\kappa}$. This means that $V$ has only $\kappa$ many grounds.

By Corollary 5.5, Proposition 6.1, and Corollary 6.3, we have the following:

Corollary 6.4. Suppose $\kappa$ is a hyper-huge cardinal. Then the following hold: 
(1) $\mathbb{M}$ is a solid bedrock of all forcing extensions of $V$.

(2) There is a poset $\mathbb{P} \in \mathbb{M}_{\kappa}$ and a $(\mathbb{M}, \mathbb{P})$-generic $G$ with $V=\mathbb{M}[G]$.

(3) $V$ has only $<\kappa$ many grounds.

Proof. (1) and (2) are immediate from Proposition 6.1, Corollaries 5.5, and 6.3.

For (3), fix a poset $\mathbb{P} \in \mathbb{M}_{\kappa}$ and a $(\mathbb{M}, \mathbb{P})$-generic $G$ with $V=\mathbb{M}[G]$. By Fact 3.5 , every ground $W$ of $V$ is of the form $\mathbb{M}[H]$ for some $H \subseteq \mathbb{Q} \subseteq \operatorname{ro}(\mathbb{P})^{\mathbb{M}}$. Hence there are only $2^{|r o(\mathbb{P})|}(<\kappa)$ many grounds of $V$.

As mentioned before, $\mathbb{M}$ is a model of $\mathrm{ZFC}+$ the Ground Axiom if $\mathbb{M}$ is a ground. If $\kappa$ is hyper-huge, then $\mathbb{M}$ is a ground of $V$ and $V$ is a small forcing extension of $V$ relative to $\kappa$. Then one can check $\kappa$ remains hyper-huge in $\mathbb{M}$ as follows: Suppose $V=\mathbb{M}[G]$ for some $G \subseteq \mathbb{P} \in \mathbb{M}$ with $|\mathbb{P}|<\kappa$. For a given $\lambda \geq \kappa$, let $j: V \rightarrow N$ be an elementary embedding with critical point $\kappa$ and ${ }^{j(\lambda)} N \subseteq N$. $j \uparrow \mathbb{M}$ is an elementary embedding from $\mathbb{M}$ to $\mathbb{M}^{N}$. $\mathbb{M}$ satisfies the $\mu$-covering and the $\mu$-approximation properties for $V$ for some $\mu<\kappa$ by Fact 3.5. By the results in Hamkins [5], we know that $j \uparrow \mathbb{M}$ and $\mathbb{M}^{N}$ are definable in $\mathbb{M}$, and $\mathbb{M}^{N}$ is closed under $j(\lambda)$-sequences in $\mathbb{M}$. $\lambda$ was arbitrary, hence we have that $\kappa$ is hyper-huge in $\mathbb{M}$. Thus $\mathbb{M}$ is a model of $\mathrm{ZFC}+$ the Ground Axiom+hyper-huge cardinal exists.

Proposition 6.1 also shows the destructibility phenomenon of hyper-huge cardinals. Laver [9] proved that a supercompact cardinal $\kappa$ can be indestructible for $<\kappa$-directed closed forcings. In contrast to Laver's theorem, Bagaria-HamkinsTsaprounis-Usuba [1] showed that, if $\kappa$ is extendible, then every non-trivial $<\kappa$ closed forcing must destroy the extendability of $\kappa$. Now, by Proposition 6.1, we know that any hyper-huge cardinals must be destroyed by any non-trivial non-small forcings. The following is just a rephrasing of Proposition 6.1:

Corollary 6.5. Let $\kappa$ be an infinite cardinal. Let $\mathbb{P}$ be a poset, and suppose for every $p \in \mathbb{P}$, the suborder $\{q \in \mathbb{P}: q \leq p\}$ is not forcing equivalent to a poset of size $<\kappa$. Then $\mathbb{P}$ forces that " $\kappa$ is not hyper-huge".

\section{Some CONSEQUEnCES OF MAIn THEOREMS}

In this section, we discuss some consequences of our main theorems.

The generic HOD, gHOD, is the intersection of HOD of all forcing extensions of $V$ (Fuchs-Hamkins-Reitz [4]). gHOD is definable as the class $\bigcap\left\{\operatorname{HOD}^{V^{\operatorname{Col}(\alpha)}}: \alpha\right.$ is an ordinal\}.

Proposition 7.1. HOD ${ }^{\mathbb{M}}$ is a subclass of gHOD. If $\mathbb{M}$ is a ground of $V$ then $\mathrm{HOD}^{\mathrm{M}}$ coincides with $\mathrm{gHOD}$.

Proof. First note that, by Corollary 5.5, we know that $g \mathbb{M}=\mathbb{M}$, hence $\operatorname{HOD}^{g \mathbb{M}}=$ $\mathrm{HOD}^{\mathbb{M}}$. 
We show that $\mathrm{HOD}^{\mathbb{M}}=\mathrm{HOD}^{g \mathbb{M}} \subseteq$ gHOD. Take an arbitrary generic extension $V[G]$ of $V$. It is enough to show that $\mathrm{HOD}^{g \mathbb{M}} \subseteq \mathrm{HOD}^{V[G]}$. Since $g \mathbb{M}$ is a parameter free definable class in $V[G]$, we have that $g \mathbb{M}_{\alpha} \in \mathrm{OD}^{V[G]}$ for every $\alpha$. This means that $\operatorname{HOD}^{\mathbb{M}}=\operatorname{HOD}^{g \mathbb{M}} \subseteq \operatorname{HOD}^{V[G]}$.

Next suppose $\mathbb{M}$ is a ground of $V$. Put $V=\mathbb{M}[G]$ for some $G \subseteq \mathbb{P} \in \mathbb{M}$. Then we can find an ordinal $\theta$ greater than $\left|2^{|\mathbb{P}|}\right|^{\mathbb{M}}$, an $(\mathbb{M}, \operatorname{Col}(\theta))$-generic $H$, and a $(V, \operatorname{Col}(\theta))$-generic $H^{\prime}$ such that $V\left[H^{\prime}\right]=\mathbb{M}[H]$, where $\operatorname{Col}(\theta)$ is a standard forcing adding a surjection from $\omega$ onto $\theta \cdot \operatorname{Col}(\theta)$ is weakly homogeneous, so we have that $\operatorname{HOD}^{V^{\operatorname{Col}(\theta)}}=\operatorname{HOD}^{\mathbb{M}[H]} \subseteq \mathrm{HOD}^{\mathbb{M}}$. $\mathrm{gHOD} \subseteq \mathrm{HOD}^{V^{\operatorname{Col}(\theta)}}$, thus we have $\mathrm{gHOD} \subseteq \mathrm{HOD}^{V \operatorname{Col}(\theta)} \subseteq \mathrm{HOD}^{\mathbb{M}}$.

Note 7.2. If a hyper-huge cardinal exists, then $\mathbb{M}$ is a ground of $V$ by Corollary 6.4. Combining this observation with Proposition 7.1, we have that, if a hyper-huge cardinal exists, then $\mathrm{HOD}^{\mathbb{M}}$ must coincide with gHOD.

Note 7.3. We also note that $\mathrm{HOD}^{\mathrm{M}} \subsetneq$ gHOD is possible. We use the following fact:

Fact 7.4 (Fuchs-Hamkins-Reitz [4]). There is a class forcing extension $V[G]$ in which $V=\mathbb{M}^{V[G]}=g \mathbb{M}^{V[G]}=\mathrm{gHOD}^{V[G]}$.

Starting with a model $\operatorname{HOD}^{V} \subsetneq V$, take a class forcing extension $V[G]$ in which $V=\mathbb{M}^{V[G]}=\operatorname{gHOD}^{V[G]}$. Then $\operatorname{HOD}^{\mathbb{M}^{V[G]}}=\operatorname{HOD}^{V} \subsetneq V=\operatorname{gHOD}^{V[G]}$.

The following gives some restrictions on $V[G]$ in the above fact.

Proposition 7.5. If $V \neq \mathbb{M}$, then, for every outer transitive model $W \supseteq V$ of $Z F C$, if $\mathbb{M}^{W}$ is a ground of $W$, then $\mathbb{M}^{W} \neq V$ (so $g \mathbb{M}^{W} \neq V$ ). In particular, if $W$ has a hyper-huge cardinal, then $\mathbb{M}^{W} \neq V$.

Proof. Take an outer model $W \supseteq V$. By our assumption, $\mathbb{M}^{W}$ is a solid bedrock of $W$ by Corollary 5.5. Since $V \neq \mathbb{M}^{V}$, we have that $V$ has a proper ground $M$. If $V=\mathbb{M}^{W}$, then $M$ is a ground of $\mathbb{M}^{W}$, so $M=\mathbb{M}^{W}=V$ by the minimality of $\mathbb{M}^{W}$. This is a contradiction.

Next we turn to the generic multiverse, which was first introduced in Woodin [16]. In [16], Woodin defined his generic multiverse as follows. For a given countable transitive model $M_{0}$ of ZFC, the generic multiverse of $M_{0}$ is a collection $\mathcal{F}$ of countable transitive models of ZFC such that:

(1) $M_{0} \in \mathcal{F}$.

(2) If $M \in \mathcal{F}$ and $W \subseteq M$ is a ground of $M$, then $W \in \mathcal{F}$.

(3) If $M \in \mathcal{F}, \mathbb{P} \in M$ is a poset, and $G$ is $(M, \mathbb{P})$-generic, then $M[G] \in \mathcal{F}$.

(4) $\mathcal{F}$ is a minimal collection satisfying (1)-(3).

Note that (4) is equivalent to the following $\left(4^{\prime}\right)$ : 
(4') For every $M, N \in \mathcal{F}$, there are finitely many $W_{0}, \ldots, W_{n} \in \mathcal{F}$ such that $W_{0}=M, W_{n}=N$, and, for each $i<n, W_{i}$ is a ground or a forcing extension of $W_{i+1}$.

Note also that if $M_{0}$ is fixed, then Woodin's generic multiverse is unique for $M_{0}$, so it is the generic multiverse of $M_{0}$.

On the other hand, Steel [14] gave a slightly different definition of the generic multiverse. For a transitive set model $M_{0}$ of ZFC, a generic multiverse of $M_{0}$ is a collection $\mathcal{F}$ of transitive models of $\mathrm{ZFC}$ such that:

(1) $M_{0} \in \mathcal{F}$.

(2) If $M \in \mathcal{F}$ and $W \subseteq M$ is a ground of $M$, then $W \in \mathcal{F}$.

(3) If $M \in \mathcal{F}$ and $\mathbb{P} \in M$ is a poset, then there is an $(M, \mathbb{P})$-generic $G$ with $M[G] \in \mathcal{F}$.

(4) If $M, N \in \mathcal{F}$, then there is $W \in \mathcal{F}$ which is a common forcing extension of $M$ and $N$.

Unlike Woodin's generic multiverse, Steel's generic multiverse is not unique for $M_{0}$; it is possible that $M_{0}$ has many Steel's generic multiverses.

To treat Woodin's and Steel's generic multiverses simultaneously, we adopt the following definition of generic multiverse, which is weaker than Woodin's and Steel's definitions.

Definition 7.6. For a transitive set model $M_{0}$ of ZFC, a generic multiverse of $M_{0}$ is a collection $\mathcal{F}$ of transitive models of ZFC such that:

(1) $M_{0} \in \mathcal{F}$.

(2) If $M \in \mathcal{F}$ and $W \subseteq M$ is a ground of $M$, then $W \in \mathcal{F}$.

(3) If $M \in \mathcal{F}$ and $\mathbb{P} \in M$ is a poset, then there is an $(M, \mathbb{P})$-generic $G$ with $M[G] \in \mathcal{F}$.

(4) For every $M, N \in \mathcal{F}$, there are finitely many $W_{0}, \ldots, W_{n} \in \mathcal{F}$ such that $W_{0}=M, W_{n}=N$, and, for each $i<n, W_{i}$ is a ground or a forcing extension of $W_{i+1}$.

Note that if $M_{0}$ has a generic multiverse in our sense, then $M_{0}$ must be countable or $M_{0} \cap O N=\omega_{1}$. Our definition of generic multiverse is weak, so if $\mathcal{F}$ is a generic multiverse in the sense of Woodin or Steel, then it also is in our sense.

Now fix a transitive model $M_{0}$ of ZFC with $M_{0} \cap O N \leq \omega_{1}$.

Lemma 7.7. If $\mathcal{F}$ is a generic multiverse of $M_{0}$, then every two members of $\mathcal{F}$ have a common ground.

Proof. Take $M, N \in \mathcal{F}$. By the definition of $\mathcal{F}$, there are finitely many $W_{0}, \ldots, W_{n} \in$ $\mathcal{F}$ such that $W_{0}=M, W_{n}=N$, and, for each $i<n, W_{i}$ is a ground or a forcing extension of $W_{i+1}$. By induction on $i<n$, we show that $W_{0}$ and $W_{i+1}$ have a common ground. The case $i=0$ is clear. Suppose $W_{0}$ and $W_{i}$ have a common 
ground $W$. If $W_{i}$ is a ground of $W_{i+1}$, then $W$ is a common ground of $W_{0}$ and $W_{i+1}$. Suppose $W_{i}$ is a forcing extension of $W_{i+1}$. Then $W$ and $W_{i+1}$ are grounds of $W_{i}$. By the DDG of $W_{i}, W$ and $W_{i+1}$ have a common ground $W^{\prime}$, which is a common ground of $W_{0}$ and $W_{i+1}$.

This lemma shows that, in Definition 7.6, under the presence of (1)-(3), the clause (4) can be replaced by "every two members of $\mathcal{F}$ have a common ground".

Proposition 7.8. Let $\mathcal{F}$ be a generic multiverse of $M_{0}$. Then $\mathcal{F}$ is just the collection

$\left\{M \in \mathcal{F}: M\right.$ is a forcing extension of some ground of $\left.M_{0}\right\}$.

Moreover, for $M, N \in \mathcal{F}$, if $M \subseteq N$, then $M$ is a ground of $N$.

Proof. Let $\mathcal{F}^{\prime}=\left\{M \in \mathcal{F}: M\right.$ is a forcing extension of some ground of $\left.M_{0}\right\}$. It is clear that $\mathcal{F}^{\prime} \subseteq \mathcal{F}$. If $M \in \mathcal{F}$, then $M$ and $M_{0}$ have a common ground $W \in \mathcal{F}$ by Lemma 7.7. Then $M \in \mathcal{F}^{\prime}$ by the definition.

Next take $M, N \in \mathcal{F}$ with $M \subseteq N$. By Lemma 7.7 again, $M$ and $N$ have a common ground $W$. Since $N$ is a forcing extension of $W$ and $W \subseteq M \subseteq N, N$ is a forcing extension of $M$ by Fact 3.1.

If $\mathbb{M}$ is a ground, then every ground is a forcing extension of $\mathbb{M}$. Hence we have the following simple view of a generic multiverse under this assumption:

Proposition 7.9. Suppose $\mathbb{M}^{M_{0}}$ is a ground of $M_{0}$. Then for every generic multiverse $\mathcal{F}$ of $M_{0}$, the following hold:

(1) $\mathbb{M}^{M_{0}}=\mathbb{M}^{N}$ for every $N \in \mathcal{F}$.

(2) $\mathbb{M}^{M_{0}}$ is the minimum member of $\mathcal{F}$.

(3) Every member of $\mathcal{F}$ is a forcing extension of $\mathbb{M}^{M_{0}}$.

Let $\mathcal{F}$ be a generic multiverse of $M_{0}$. A sentence $\varphi$ of set-theory is a multiverse truth (on $\mathcal{F}$ ) if every member of a generic multiverse $\mathcal{F}$ satisfies $\varphi$. Woodin [16] showed that there is a computable translation ()$^{*}$ on sentences such that, for every sentence $\varphi, \varphi$ is a multiverse truth if and only if $(\varphi)^{*}$ holds in $M_{0}$. By the DDG, we have the following simple translation. The next proposition is immediate from Propositions 7.8 and 7.9 .

Proposition 7.10. Let $\mathcal{F}$ be a generic multiverse of $M_{0}$, and $\varphi$ a sentence of set theory.

(1) $\varphi$ is a multiverse truth if and only if, for every ground $W$ of $M_{0}$ and every poset $\mathbb{P} \in W, \mathbb{P}$ forces $\varphi$ in $W$.

(2) If $\mathbb{M}^{M_{0}}$ is a ground of $M_{0}$, then $\varphi$ is a multiverse truth if and only if, for every poset $\mathbb{P} \in \mathbb{M}^{M_{0}}, \mathbb{P}$ forces $\varphi$ in $\mathbb{M}^{M_{0}}$.

We also have the following: 
Proposition 7.11. Let $\mathcal{F}$ be a generic multiverse of $M_{0}$, and $\varphi$ a sentence of set theory. Then $\varphi$ is a multiverse truth if and only if, for every poset $\mathbb{P} \in M_{0}, \mathbb{P}$ forces "every ground satisfies $\varphi$ " in $M_{0}$.

Proof. By the definition of a generic multiverse, if $\varphi$ is a multiverse truth then every poset forces "every ground satisfies $\varphi$ " in $M_{0}$.

For the converse, suppose there is $M \in \mathcal{F}$ such that $\varphi$ fails in $M$. By Lemma 7.7, $M$ and $M_{0}$ have a common ground $N \in \mathcal{F}$. Since $M$ is a forcing extension of $N$, there is a poset $\mathbb{P} \in N$ which forces $\neg \varphi$ in $N$. Fix a large $\theta>2^{|\mathbb{P}|}$. We can find an $\left(M_{0}, \operatorname{Col}(\theta)\right)$-generic $G$ with $M_{0}[G] \in \mathcal{F}$. In $M_{0}[G]$, there is an $(N, \mathbb{P})$-generic $H \in M_{0}[G]$. Then $N \subseteq N[H] \subseteq M_{0}[G] . \varphi$ fails in $N[H]$. Since $N$ is a ground of $M_{0}$ and $M_{0}$ is a ground of $M_{0}[G], N[H]$ is a ground of $M_{0}[G]$. This means that $\operatorname{Col}(\theta)$ forces that "there is a ground which does not satisfy $\varphi$ " in $M_{0}$.

Note 7.12. If a hyper-huge cardinal exists, then $\mathbb{M}$ is a ground of $V$. Hence, if a hyper-huge cardinal exists in $M_{0}$, then the conclusions of Proposition 7.9 and of (2) in Proposition 7.10 hold.

Finally we discuss the maximality principles, that were studied by Hamkins [6]. Let $\varphi(x)$ be a formula of set theory with free variable $x$, and $s$ a set. A sentence $\varphi(s)$ is forceable if there is a poset $\mathbb{P}$ which forces $\varphi(s)$, and $\varphi(s)$ is necessary if every poset $\mathbb{P}$ forces $\varphi(s)$. The Maximality principle, MP, is the assertion that, every forceably necessary sentence is true in $V$, that is, for every sentence $\varphi$, if

$$
\exists \mathbb{P}\left(\Vdash_{\mathbb{P}} " \forall \mathbb{Q} \Vdash_{\mathbb{Q}} \varphi "\right),
$$

then $\varphi$ holds in $V . \mathrm{MP}(\mathbb{R})$ is the assertion that, for every formula $\varphi(x)$ and $r \in \mathbb{R}$, if $\varphi(r)$ is forceably necessary, then $\varphi(r)$ holds in $V$. $\square \mathrm{MP}(\mathbb{R})$ is the assertion that $\operatorname{MP}(\mathbb{R})$ is necessary, that is, every poset $\mathbb{P}$ forces that $\operatorname{MP}\left(\mathbb{R}^{V^{\mathbb{P}}}\right)$ holds.

Hamkins [6] showed that MP is equiconsistent with $\mathrm{ZFC}$, and that $\mathrm{MP}(\mathbb{R})$ is consistent relative to some large cardinal assumption which is weaker than a Mahlo cardinal. Moreover, by the proofs in [6], MP and even $\mathrm{MP}(\mathbb{R})$ are consistent with almost all large cardinals. Woodin proved that $\square \mathrm{MP}(\mathbb{R})$ is consistent relative to some large cardinal assumption. However, Woodin's model is an extension of some canonical model of $\mathrm{AD}_{\mathbb{R}}$, and no strong large cardinals exist in the resulting model. Hence it is natural to ask if $\square \mathrm{MP}(\mathbb{R})$ is consistent with strong large cardinals, for example, supercompact cardinals. Now we can show that our hyper-huge cardinal is inconsistent with $\square \mathrm{MP}(\mathbb{R})$.

Proposition 7.13. If $\square \mathrm{MP}(\mathbb{R})$ holds, then $\mathbb{M}$ is not a ground of $V$. In particular, if there exists a hyper-huge cardinal, then $\square \mathrm{MP}(\mathbb{R})$ fails.

The proof is immediate from Corollary 5.5 and the following fact: 
Fact 7.14 (Hamkins [6]). Suppose $\square \mathrm{MP}(\mathbb{R})$. Let $M \subseteq V$ be a forcing invariant parameter free definable transitive model of ZFC. Then, for every infinite ordinal $\alpha,\left(\alpha^{+}\right)^{M}$ is not a cardinal in $V$. In particular, if $\square \mathrm{MP}(\mathbb{R})$ holds, then, for every infinite ordinal $\alpha,\left(\alpha^{+}\right)^{\mathbb{M}}$ is not a cardinal in $V$.

Acknowledgements: We would like to thank Joel David Hamkins and Daisuke Ikegami for many fruitful discussions. Some of the ideas that came out of those discussions were used in this paper. We also thank Paul Larson and Yo Matsubara, they gave the author many corrections of the draft and insightful suggestions. Finally we are grateful to David Aspero, Hiroshi Sakai, Kostantinos Tsaprounis, Yasuo Yoshinobu for their useful comments. This research was supported by KAKENHI grant Nos. 15K17587 and 15K04984.

\section{REFERENCES}

[1] J. Bagaria, J. D. Hamkins, K. Tsaprounis, T. Usuba, Superstrong and other large cardinals are never Laver indestructible. Arch. Math. Logic, 55 (2016), no. 1-2, 19-35.

[2] L. Bukovský, Characterization of generic extensions of models of set theory. Fund. Math. 83 (1973), no. 1, 35-46.

[3] S. D. Friedman, S. Fuchino, H. Sakai, On the set-generic multiverse. Proceedings of the Singapore Programme on Sets and Computations, to appear.

[4] G. Fuchs, J. D. Hamkins, J. Reitz, Set-theoretic geology. Ann. Pure Appl. Logic 166 (2015), no. $4,464-501$.

[5] J. D. Hamkins, Extensions with the approximation and cover properties have no new large cardinals. Fund. Math. 180 (2003), no. 3, 257-277.

[6] J. D. Hamkins, A simple maximality principle. J. Symbolic Logic 68 (2003), no. 2, 527-550.

[7] T. Jech, Set theory. The third millennium edition, revised and expanded. Springer-Verlag, 2003.

[8] D. R. Kurepa, Ensembles ordonnés et ramifieś. Publ. Math. Univ. Belgrade 4 (1935), 1-138.

[9] R. Laver, Making the supercompactness of $\kappa$ indestructible under $\kappa$-directed closed forcing. Israel J. Math. 29 (1978), no. 4, 385-388.

[10] R. Laver, Certain very large cardinals are not created in small forcing extensions. Ann. Pure Appl. Logic 149 (2007), no. 1-3, 1-6.

[11] W. J. Mitchell, On the Hamkins approximation property. Ann. Pure Appl. Logic 144 (2006), no. $1-3,126-129$.

[12] J. Reitz, The Ground Axiom. J. of Symbolic Logic 72 (2007), no. 4, 1299-1317.

[13] R. Schindler, The long extender algebra. Archive for Math. Logic., to appear.

[14] J. Steel, Gödel's program. in: Interpreting Gödel Critical Essays, Cambridge University Press, 2014.

[15] T. Usuba, The approximation property and the chain condition. RIMS Kokyuroku, No. 1985 (2014), 130-134.

[16] W. H. Woodin, The continuum hypothesis, the generic-multiverse of sets, and the $\Omega$ conjecture. Set theory, arithmetic, and foundations of mathematics: theorems, philosophies, 13-42, Lect. Notes Log., 36, Assoc. Symbol. Logic, La Jolla, CA, 2011.

[17] A. Zarach, Unions of $Z F^{-}$-models which are themselves $Z F^{-}$-models. Logic Colloquium '80 (Prague, 1980), pp. 315-342, Stud. Logic Foundations Math., 108, North-Holland 1982. 
[18] A. Zarach, Replacement $\nrightarrow$, Collection. In: Gödel '96 (Brno, 1996), Lecture Notes Logic Vol. 6, pp. 307-322. Springer, 1996.

(T. Usuba) Faculty of Science and Engineering, Waseda University, Okubo 3-4-1, SHINJYUKU, TOKYO, 169-8555 JAPAN

E-mail address: usuba@waseda.jp 